\title{
Innovative Strategy to Meet the Challenges of the Future Digital Society
}

\author{
Olivier Bonnaud* \\ Department of Sensors and Microelectronics, IETR, University of Rennes 1, 35042 Rennes, France \\ National Coordination for Education in Microelectronics, GIP-CNFM, 38016 Grenoble, France \\ Received 12 November 2020; received in revised form 30 December 2020; accepted 04 February 2021 \\ DOI: https://doi.org/10.46604/aiti.2021.6724
}

\begin{abstract}
Today, we are experiencing a societal revolution with the development of digital technologies, and it brings new challenges. Indeed, the number of connected objects, intelligent sensors and IoTs are increasing exponentially. The same goes for the resulting energy consumption. Beyond 2030, without a radical transformation of communication technologies and protocols, the digital world will be at an energy dead end. All these objects are physically realized with microelectronic devices and systems. This analysis of the microelectronics community has led the French government to recognize an electronics sector that is becoming a priority area of industrial policy. The Strategic Committee of this sector has proposed innovations applied to the entire digital chain including all facets of the microelectronics field and human skills and know-how. The technological and energy issues are thus presented, and the proposed solutions were addressed. They concern both technological and human aspects. This paper ends by giving examples of the implementation of innovative approaches which essentially include the electronic functions involved in connected objects and which are intended to bring the know-how of future actors in the field.
\end{abstract}

Keywords: digital society, microelectronics, multidisciplinarity, energy challenge, higher education training

\section{Introduction}

It is globally recognized that we are experiencing a societal revolution with the development of digital technologies, connected objects and the Internet of Things (IoT) [1]. While this rapid evolution may seem easy and problem-free, in reality, it raises new and extremely important challenges. Indeed, the number of connected objects, smart sensors, Artificial Intelligence systems (AI) and IoTs is growing exponentially. All these objects, functions and systems take part in the Industrial Revolution 4.0. [2], which will involve many robots, collaborative robots [3], connected monitoring, and management. As a consequence, the resulting energy consumption of this digital world is growing exponentially as well. All of these objects and systems are physically realized using microelectronic and nanotechnological devices, circuits and systems, as well as smart sensors and actuators, opening the way to multidisciplinary applications [4]. Beyond 2030, without a radical transformation of technologies and communication protocols, the digital world will be in an energy dead-end and most likely also in a digital security dead-end [5].

This analysis by the microelectronics community has led to the French government's recognition of an electronics sector that is becoming a priority axis of industrial policy [6]. A Strategic Sector Committee has been set up, bringing together industry representatives, researchers and academic forces in the field of electronics and microelectronics. It acts as a structure for brainstorming and positions itself as a force for proposals to government ministries. It highlights innovative topics applied to the entire digital chain and which are part of the technical challenges. They will be applied to elementary components and to the architectures of electronic circuits and systems for the detection, processing and storage of information as well as signal

* Corresponding author. E-mail address: olivier.bonnaud@univ-rennes1.fr 
transmission. If these topics seem to be part of a classical approach, in practice the concepts to be implemented are new. Indeed, designers and manufacturers have tried in the past to bring new products with new functions to the market as quickly as possible, regardless of energy consumption and environmental impact. Their approach has been to implement the existing product using IP (intellectual property) and through improved integration, the size of the circuits and systems has not increased. In addition, in order to produce faster and cheaper, many companies have ceased their activities and relocated the manufacture of electronic devices and systems abroad, particularly, to the Far East. While this approach seems very good economically, in the long term it means a loss of skills and foreign dependency that can affect strategic sectors. This evolution generates new challenges that lead to a new strategy. This strategy must respond to an increase in human skills and know-how, both qualitatively and quantitatively, in order to meet these challenges.

The main objective of this work is to describe the strategy adopted by the French industrial electronics sector and by the national academic network in charge of higher education in the field of electronics, microelectronics and nanotechnologies, in order to meet the challenges on both levels. The first one deals with the technological aspect with an innovative approach that particularly decreases the power consumption of all IoT applications. Note that the new connected objects are designed to save energy in many societal applications related to transportation or smart home for example [4]. The second one is the human aspect in the framework of improving knowledge and know-how in order to reduce the shortage of skills in this exponentially growing field. Future actors will have to ensure innovation in connected objects and their applications in all sectors of society.

Thus, this document focuses successively on the strategy set-up by the microelectronics industry sector in France, on the detailed description of the technological and energy challenges that highlight human needs, and finally on the actions carried out within the national training network in microelectronics and nanotechnologies to train future actors in this field. Special attention is paid to the current contribution to training and to the innovation strategy that needed to meet future challenges.

\section{Digital Society and Microelectronics Links}

In order to fully understand the importance of the strategy and the challenges in the field of microelectronics, this first part deals with the situation of the field in the context of the future digital society. This evolution is global. However, in order to describe concrete actions, the case of France is highlighted.

\subsection{Dependence of the digital society on the electronics sector}

The revolution of smart and connected objects coupled with the energy transition is affecting all sectors of industry and the growth of the next few decades will belong to those who can benefit from these technological innovations based on electronics [6]. Electronics is the essential industrial base for the production of digital equipment and systems. The electronics industries thus take a central place in the French industrial landscape and are at the heart of the digital transformation [7]. After several years of reflection and consultation, the electronics industry sector was found on March 15, 2019 by the General Directorate of Enterprises within the Ministry in charge of Economy, Finance and Industry [8].

The "electronic sector" is therefore at the heart of the societal evolution towards digital and connected objects (IoT, Internet of Things, Internet of Every-thing!). In France, it represents 1,100 companies and 230,000 jobs for a turnover of 15 billion Euros [6]. In order to meet the challenges, this sector must master the 4 complementary pillars which are:

(1) Technologies and electronic components including intelligent sensors to create data,

(2) The connected objects to process, transmit and develop the associated services,

(3) Power electronics to support the energy transition and the development of electric mobility,

(4) Cybersecurity to build the confidence necessary for the development of electronic technologies in industry (cyber-physical systems). 


\subsection{Major role of microelectronics, micro- and nanotechnologies}

Within this sector, microelectronics occupies a central place since all the objects involved in smart and connected objects are made up of electronic components, circuits and systems. The great majority of which are integrated and carry embedded software that provides the intelligence of the application devices. In addition, many new systems include passive devices and micromechanical elements for sensing or actuations. To resume, the connected objects are primarily made up of multiple microelectronic, microtechnological and communications devices [9] that are judiciously combined in a full system.

This evolution has been made possible by the fabulous growth of microelectronic integration over the last sixty years. The number of elementary components in an integrated circuit has increased from a few units to a few billion thanks to the reduction of lateral dimensions in a plane [10]. Unfortunately, this reveals a physical limitation related to the distance between atoms and the quantum effects attached to them. But about fifteen years, this limitation has been overcome by playing on the third dimension [11], which makes it possible to have integrated systems of several thousand functions that can each contain billions of elementary components [12]. These results make it possible to have data processing systems in fabulous quantities in ever shorter times and have paved the way for connected objects, IoT, AI and the Industrial Revolution 4.0. All these aspects are taken into consideration in the strategy at the national level.

\subsection{Main objectives resulting from the analysis of the French electronics sector}

The French electronics sector has established a strategic committee composed mainly of industrial members but also of academic members. The main goal of this strategic committee is to develop activities capable of meeting the challenges mentioned above. The committee has thus identified four key priorities in its work:

(1) Maintaining excellence in key digital technologies by increasing R\&D\&I efforts and developing strategic partnerships;

(2) Adapting skills by anticipating changing needs (employment and skills) and by developing work-linked training offer;

(3) Promoting competitive "Made in France" electronics manufacturing;

(4) Positioning the sector as a key player in the digital transformation by supporting SMEs in other sectors.

Within the framework of these priorities, the strategic committee set up four working groups in 2019 that produced several documents proposing priority actions and objectives. The first priority directly concerns the purely technological aspects with the associated constraints and challenges. It mainly concerns the policy towards research laboratories and research development departments of companies. The second priority relates to the adaptation of skills and know-how, which presents a challenge in terms of training content at all levels and the number of people trained. Everything possible must be done to achieve these objectives that are:

(1) Raising young people's awareness of the field in order to make it more attractive,

(2) Initial training from technician to engineer level,

(3) Advanced training through research to develop innovation,

(4) Life-long training to enable the company's employees to adapt to innovative technologies. It is clear that we will focus on these two priorities that directly involve universal strategy and actions. To this end, it is useful to detail the different areas of scientific and technical expertise and know-how. In other words, we need to highlight the technical and technological Connected Objects and Challenges 


\section{Connected Objects and Challenges}

The digital society and future Industry 4.0 are based on internet of things (IoT). In terms of physical support, the basic system is the connected object that gathers many electronic functions. In addition, the connected object fills also a mission of control, monitoring processes or robots. In order to well select the skills and the orientation of the training, it is useful to highlight the several associated works, limitations and related challenges.

\subsection{Usual architecture of a connected object}

The first mission of a connected object is to retrieve a physical, chemical or biological quantity by means of a sensor that transforms it into an electrical signal, amplifies and treat the signal, transmits it remotely to a processing system in order to have a feedback through the whole chain that can correct the original quantity. It is thus composed of an on-site part, a remote part and a communication system between the two. The connected objects must perform functions such as detecting a physical, chemical or biological analogue signal, transforming it into digital signals via converters, storing and transmitting it via communication protocols, receiving it in databases and data centers, analysis processing centers, and performing the return chain to an actuator that can control the application phenomenon. Thus, all these objects have a common architecture as shown in Fig. 1, which includes sensors and actuators, electronic modules for signal processing, data transmission and reception modules, but also devices for monitoring, visualization, alarms, memorization, energy recovery and storage, etc. [9].

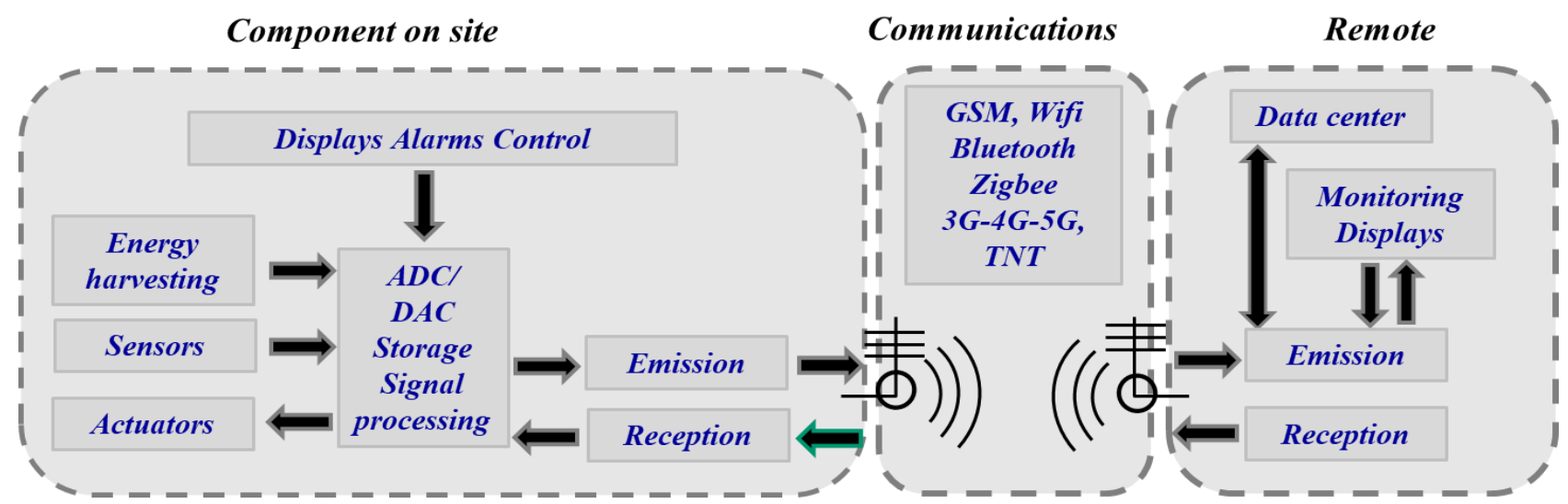

Fig. 1 Schematic architecture of a connected object. Many electronic functions are involved in the full system

\subsection{Multiplication of connected objects and sensors}

As already mentioned, the enormous integration capacity of microelectronics allows multiplying not only the connected objects, but also the sensors and the IoT [13]. The number of these functions and objects is growing exponentially, as shown in Fig. 2 [14]. It is interesting to note that the development of heterogeneous integration has led to the development of sensor design and fabrication. For example, about thirty sensors are installed in a regular cell phone, while seventy are wired in a standard car.

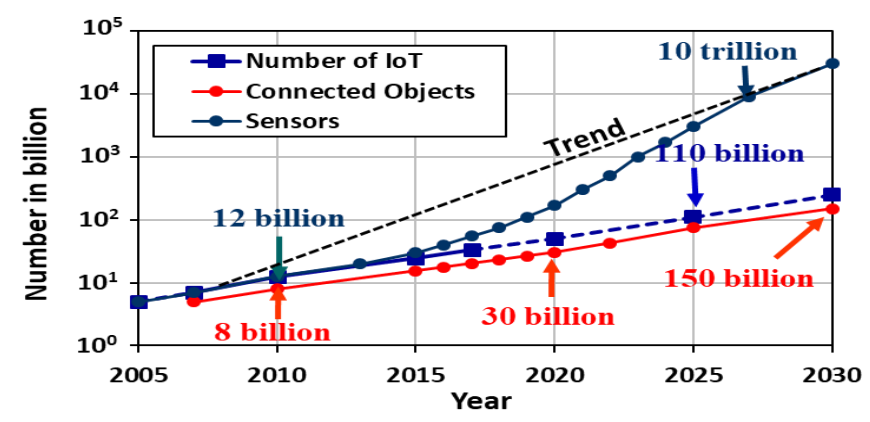

Fig. 2 Exponential growth rate of the number of IoT, connected objects and sensors; a direct effect of the microelectronics integration growth 


\subsection{Energy consumption challenges}

The consequence of this exponential growth is that the energy consumed by connected objects is also growing exponentially and is becoming globally preponderant [15] as it will be shown below. Indeed, one gigabyte of data downloaded from the Internet sums up to $5.12 \mathrm{kWh}$ [16]; loading a film online (equivalent to a 5GB DVD) consumes $25 \mathrm{kWh}$ ! According to recent literature, the contribution to this consumption is distributed as shown in Fig. 3:

\section{Distribution of consumption}

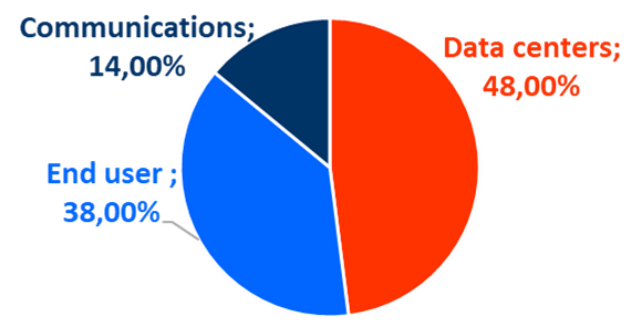

Fig. 3 Allocation of power consumption due to connected objects and IoT

$-48 \%$ by data centers (servers, routers);

- $38 \%$ by the end user (computer, smartphone, display, battery charging, screen power supply, life boxes, etc.);

- $14 \%$ for communications (cables, optical fibers, repeater-amplifiers, Hertzian or satellite transmissions).

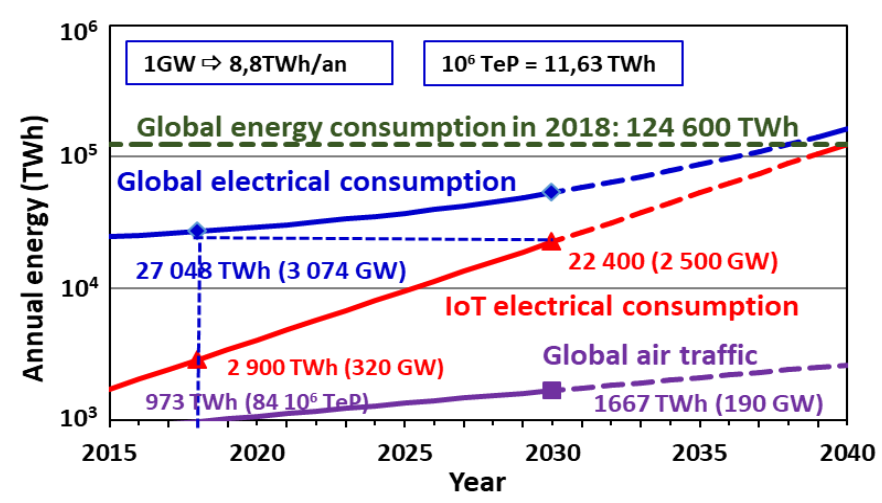

Fig. 4 Expected exponential growth of the IoT electrical energy consumption without any change in the technologies of IoT and connected objects

This distribution is approximate since some functions can be assigned to two different sectors. However, the total appears to be correct. As shown in Fig. 4 , the electricity consumption due to IoT is multiplied by 2 each 4 years. In 2018 , the electrical energy consumed by the implementation of connected objects reached 2,900 TWh, which corresponds to a permanent production of $320 \mathrm{GW}$ out of the 8,800 annual hours and represents $11 \%$ of the world's electrical energy consumption [17]. At the beginning of 2020, the consumption was equivalent to about 3 times the energy consumption of world air traffic as shown Fig. 4. Let us notice that due to the global pandemic linked to "COVID-19", the extrapolation of global air traffic beyond 2020 has been strongly affected since these curves were drawn.

Assuming current technologies are maintained, this global energy consumption could reach 124,600 TWh in 2040, which represents a permanent production of $15,000 \mathrm{GW}$ or 15,000 nuclear reactors. This energy consumption is also equivalent to the global energy production in 2018, regardless of its source and form [18]. It is clear that this situation will become unrealistic and that major changes will have to be made in the design, technology, energy consumption of connected objects, the organization of manufacturing (Industry 4.0.) and the transfer and storage of data.

In practice, the energy consumption of all physical objects will have to be considerably reduced. The first approach will consist in reducing the consumption of microelectronic devices and circuits, which account for a contribution close to $60 \%$. Assuming that the reduction gradually reaches a factor of 10 over the next 20 years, we can expect an evolution of IoT-related 
energy consumption as shown in Fig. 5. Global electrical consumption and IoT consumption in dash lines are issued of Fig. 4. Knowing that IoT consumption does not depend only on the hardware, instead of undergoing a growth in a ratio of 32 , we should limit this growth with a ratio of 6.5. The annual electrical energy consumption of IoT is expected to reach nineteen thousand terawatt-hours, which is more appropriate for the planet (full lines) and therefore more realistic. However, global consumption of electrical energy will in any case be twice as high as it is today.

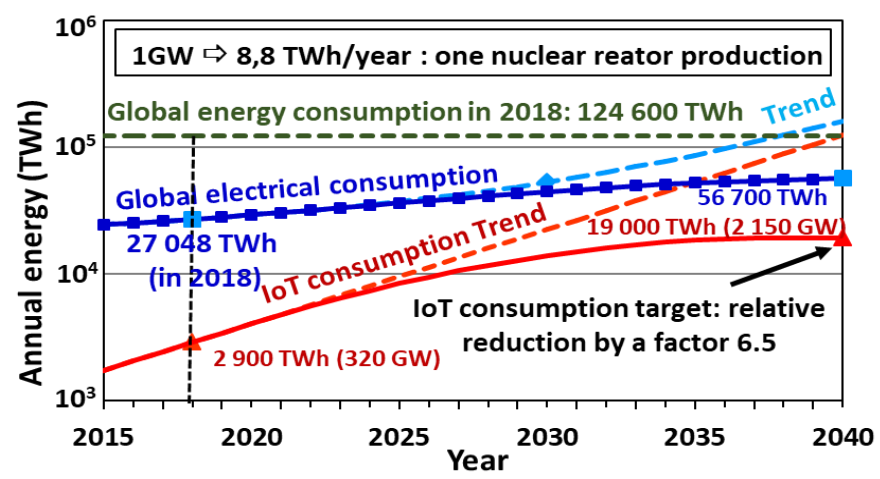

Fig. 5 Expected IoT consumption and global electrical energy consumption in 2040. Decreasing significantly the consumption of electronics devices and circuits (dash line to solid line), should allow to limit the global electrical energy consumption to 57,000 TWh a year by 2040

\subsection{Induced challenges on microelectronics technologies, integration, consumption, multidisciplinarity}

In order to achieve this reduction by a factor of 10 , many challenges will have to be met. The challenges of the discipline thus appear at different levels. The works of the French microelectronics network and of the working group of the strategic committee of the Industrial Sector have concluded on the priorities. Although already presented in a previous document [18], it is however useful to recall the enumeration of the major points [13]:

(1) The further miniaturization of elementary components in order to minimize their consumption both in operation and off-state by reducing leakage currents (tunnel effect);

(2) Improving the performance of power components and their circuits in order to reduce both switching and static losses. These properties are very important for servers and data centers which have a huge permanent energy consumption;

(3) The uses of the third dimension to improve circuit integration in order to minimize interconnection losses and improve reliability. Extensive studies will be required to control the power density to be dissipated in these three-dimensional structures;

(4) Modification of the circuit architecture to separate useful active zones from zones that can be put on standby, a way to significantly decrease the permanent energy consumption of circuits;

(5) The generalization of asynchronous control which should minimize the important losses linked to the synchronous power supply by the clocks of all the transistor control electrodes;

(6) The implementation of new low-temperature and/or large-area technologies involving new materials (inorganic or organic) for which the electrical consumption can be several orders of magnitude lower;

(7) The development of sensors and actuators in specific low-power technologies. These technologies will be able to be integrated thanks to the rapid growing heterogeneous integration, presently. Several of them will involve a multidisciplinary approach related to the application domain;

(8) Optimization of communication devices and protocols to limit frequency band occupation and data flow to keep only the useful according to the mission profile.

All these topics are introduced in the strategy of the Microelectronics industry sector and the national training network. 


\subsection{Present shortages in human skills revealed by the strategic committee}

The technical and technological challenges can only be met if the future technicians, engineers, teachers and doctors are capable of dealing with the subjects mentioned and providing innovative solutions that meet the requirements. It is therefore necessary to provide initial and continuing training adapted to this evolution and allowing them to acquire a good basic knowledge of the technique but also the know-how indispensable for design and production [19]. Due to the increasing complexity of the discipline, training must be able to cover the basic knowledge that will have to be redefined, but also the acquisition of skills in highly specialized areas. This necessitates the adaptation of study content by introducing new concepts and pedagogical approaches, including more practical learning, but also a strong awareness of multidisciplinarity in relation to the fields of application [20], project-based learning and the ability to work and innovate in a multidisciplinary team.

Currently, at the global level, companies in the electronics and microelectronics sector report a lack of applications in many skilled jobs. Training must fill this gap in the job market. This aim is in line with the missions of the French higher education training network in the field of microelectronics.

\section{The National Microelectronics Network in the Electronics Sector}

\subsection{Constitution of the CNFM network}

As already presented in the previous paper (18), this network is entitled CNFM for National Coordination for Training in Microelectronics and nanotechnologies [21], with an official structure as a GIP (for Public Interest Group). It is composed of twelve academic members who are respectively in charge of twelve common centers spread over the French territory, each center being common to several academic institutions located in the same geographic area [22]. The twelve CNFM common centers manage platforms and their technical facilities for practical hands-on training. Among the eighty technical platforms, 7 cleanrooms are mainly dedicated to Education, but also allow research and transfer activities. National services for CAD (Computer-Aided-Design) tools provided by the Montpellier CNFM common centers are dedicated to all the national community and foreign partners of the network. Two industrial unions and especially the most representative association ACSIEL Alliance Electronics consortium [23] are also the members of the CNFM body and involved in the national electronic sector policy. They represent more than 150 industrial sites.

\subsection{Concept and objective of the CNFM network}

The true essence of the GIP-CNFM network is to share technical platforms, which include technological processes, CAD, characterizations, and test. This sharing is mandatory due to the very high costs of equipment and functioning expenses. In addition, sharing pedagogical approaches between educators, and applying good practices towards students in initial training or even in Life Long Learning [24] is also a main educational target.

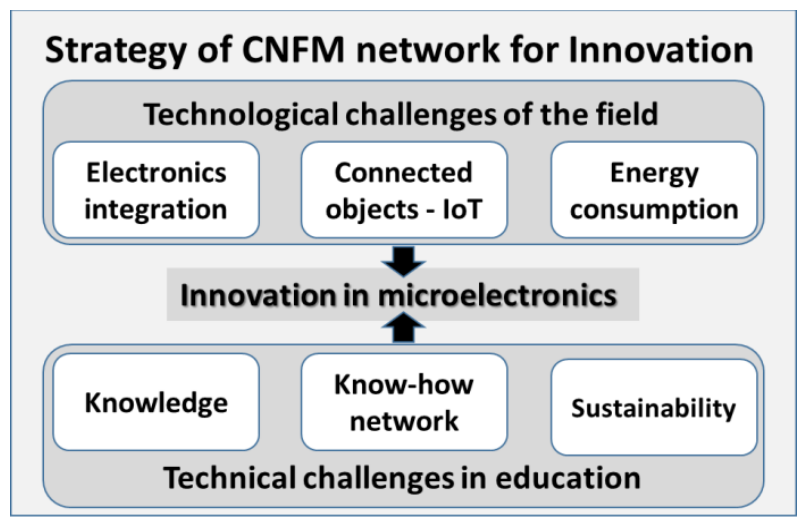

Fig. 6 The microelectronic network is at the heart of the master plan. Innovative practice enables the know-how of future players in the electronics sector to be developed 
The objective of the existing network is to provide a learning environment that allows the trainees to be immersed in a technological environment that prepares them for the industrial environment of the 21 st century and more particularly for industry 4.0. Thanks to an eight years project entitled FINMINA [25], the policy of permanent innovation in the twelve centers of the network was boosted. It allowed to develop innovative platforms with original practice all along the eight years, from 2012 to 2019 [26]. The strategy was therefore to create new practical training courses in line with the advent of the digital society. Fig. 6 summarizes the strategy to address the technological challenges while addressing the human challenges.

\subsection{Main know-how training activities}

In the majority of cases, the innovative character of the training activities stems from the research activities carried out by the teacher-researchers of the academic institutions and members of the network. This research is very often conducted in partnership with corporate research and development centers, which makes it possible to direct them towards the expressed by the sector. Fig. 7 summarizes the two indicators of the network combining skills and objectives based on an innovative approach.

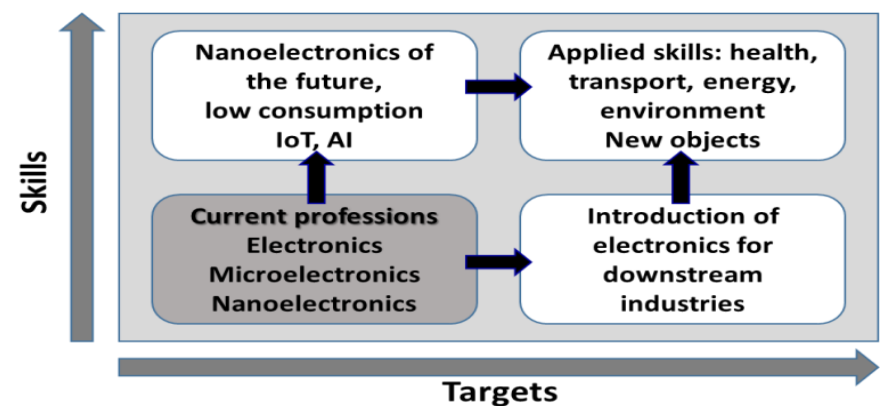

Fig. 7 Positioning of the CNFM training network in the electronics sector strategy. Innovation must improve skills in the priority actions of the electronics sector

Another point that needs to be stressed is the necessary complementarity of knowledge and know-how across the skill set [27-28]. This complementarity is more essential in the engineering disciplines and more particularly in microelectronics and whatever the technologies [29].

Various examples of platform realizations and practical work that were recently set-up by the network are given below. They address all the mentioned aspects of the evolution towards digital, knowing that a good digital technology must solve many problems related to the analogue properties of electronics! They also concern the variety of application fields, which implies the use of physical/electronic, chemical/electronic, biological/electronic transducers, which are mostly miniaturized and integrated. Subjects range from classical electronics to microelectronics and nanotechnologies, from very low power to high power, including fast communication electronics and embedded electronics, systems-on-a-chip, lab-on-a-chip, and digital security systems, which gives them a multidisciplinary aspect. Subjects range from classical electronics to microelectronics and nanotechnologies, from very low power to high power, including fast communication electronics and embedded electronics, systems-on-a-chip, lab-on-a-chip, and digital security systems, which gives them a multidisciplinary aspect [29]. These techniques can implement multi-physical design tools, integrated silicon technologies, but also technologies based on new inorganic compound and organic materials and large-area thin-film devices for display or energy harvesting [30]. Finally, they also involve digital security (in the material and equipment meaning), reliability, and testing whether electrical, physical or physical-chemical from the human to the atomic scale.

\subsection{Main results on innovative strategy of the network}

Detailed information on these activities is available on the network's website [22] and examples have already been presented in the literature according to their subject matter [28-31]. Fig. 8 shows several results of the innovative training strategy, which were provided by the network's inter-university centers. The topics concern technologies, designs, 
characterizations, sensors and systems with a multidisciplinary approach as mentioned in the main objectives. On each selected image, the name of the center and the subject are displayed. All innovative practice topics are validated by the representative of the microelectronics industry in the framework of the general assembly of the official structure of the network (GIP-CNFM), whose president is also the president of the French union of the microelectronics industry.

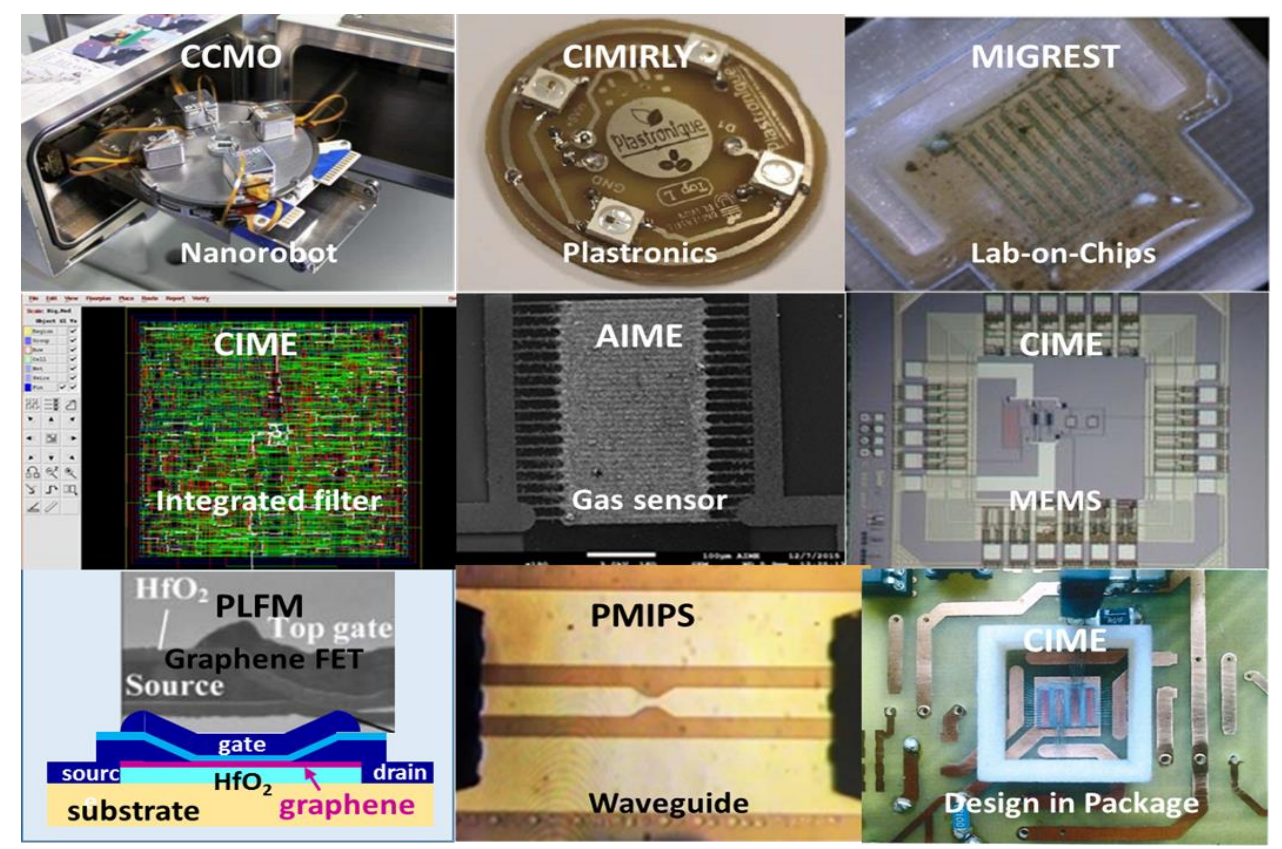

Fig. 8 Example of practice realizations by students in several network's microelectronics inter-university centers. For each picture, the name of the center and the topic are displayed

In fact, more than 80 practical and innovative learning platforms have been set up within the network over the last decade. Every year, nearly 6,000 users come to acquire know-how on these evolving platforms, with another 11,000 coming to the centers to acquire basic or awareness-raising know-how. This approach has an international interest, especially in China [32-33].

\section{Conclusions}

In an environment of continuous and tremendous technological evolution for more than 60 years, the field of microelectronics allows the development of connected objects and the advent of Industry 4.0 applied to many areas (IoT) that are experiencing exponential growth. The societal stakes are considerable, and everything must be done to limit the energy consumption of the communicating systems that will invade our daily lives. These challenges will be met thanks to high-quality, innovative training to improve the performance of the associated electronics, which must on the one hand meet these economic and societal requirements, and on the other hand be in line with the priorities of the electronics industry, which has been undergoing restructuring since 2019. The GIP-CNFM, a joint national training structure, by providing students and technical staff of industrial companies with skills and know-how in the field, is positioning itself as a major player in symbiosis with the world of industry and research, and is helping to prepare for the future by increasing the pool of trainees both qualitatively and quantitatively.

\section{Acknowledgment}

The authors want to thank all the members of the French GIP-CNFM network for they contribution to many innovative realizations. This work was financially supported by French Higher Education Ministry and by IDEFI-FINMINA program (ANR-11-IDFI-0017). A special thanks to L. Chagoya-Garzon, secretary of GIP-CNFM for her fruitful advice for the proof reading of this paper. 


\section{Conflicts of Interest}

The authors declare no conflict of interest.

\section{References}

[1] M. Burgess, "What is the Internet of Things?" WIRED Magazine, https://www.wired.co.uk/article/internet-of-things-what-is-explained-iot, February 16, 2018.

[2] A. Schütze, N. Helwig, and T. Schneider, "Sensors 4.0-Smart Sensors and Measurement Technology Enable Industry 4.0," Journal of Sensors and Sensor Systems, vol. 7, no. 1, pp. 359-371, May 2018.

[3] R. Smith, "The Future of Manufacturing: Cobots in the Factory," https://www.tctmagazine.com/tctblogs/guest-blogs/the-future-of-manufacturing-cobots-in-the-factory/, March 04, 2019.

[4] O. Bonnaud and L. Fesquet, "Microelectronics at the Heart of the Digital Society: Technological and Training Challenges," SBMicro, IEEExplore, August 2018, pp. 1-4.

[5] E. Gelenbe and Y. Caseau, The Impact of Information Technology on Energy Consumption and Carbon Emissions, ACM publication, June 2015, pp. 1-15, https://dl.acm.org/doi/pdf/10.1145/2755977.

[6] T. Tingaud, "La Filière Industries de l'Electronique," https://www.conseil-national-industrie.gouv.fr/la-filiere-industries-de-l-electronique.

[7] O. Bonnaud, "Mandatory Matching Between Microelectronics Industry and Higher Education in Engineering toward a Digital Society," Smart Education and E-Learning 2019, Springer, vol. 24, pp. 255-266, June 2019.

[8] "Signature by the Minister," https://www.economie.gouv.fr/signature-contrat-comite-strategique-filiere-industrie-electronique-plan-nano-2022.

[9] O. Bonnaud and L. Fesquet, "Innovation for Education on Internet of Things," Proceedings of Engineering and Technology Innovation, vol. 9, pp. 1-8, July 2018.

[10] G. E. Moore, "Cramming more Components onto Integrated Circuits," Electronics Magazine, vol. 38, no. 8, pp. 114-117, April 1965.

[11] K. Cheval, J. Coulm, S. Gout, Y. Layouni, P. Lombard, D. Leonard, et al., "Progress in the Manufacturing of Molded Interconnected Devices by 3D Microcontact Printing," Advanced Materials Research, vol. 1038, pp. 57-60, September 2014.

[12] R. R. Tummala and M. Swaminathan, System on Package: Miniaturization of the Entire System, 1st ed. McGraw-Hill, 2008.

[13] O. Bonnaud, "New Approach for Sensors and Connecting Objects Involving Microelectronic Multidisciplinarity for a Wide Spectrum of Applications," International Journal of Plasma Environmental Science and Technology, vol. 10, no. 2, pp. 115-120, December 2016.

[14] J. Bryzek, "The Trillion Sensors (TSensors) Foundation for the IoT," https://www.iot-inc.com/wp-content/uploads/2015/11/2-Janusz.pdf, 2015.

[15] Enerdata, "Global Energy Statistical Yearbook 2019," https://yearbook.enerdata.net/total-energy/world-energyproduction.html, March 2019.

[16] M. Greenfield, "Internet Des Objets (IoT): Nombre D'appareils Connectés Dans Le Monde De 2015 à 2025," https://fr.statista.com/statistiques/584481/internet-des-objets-nombre-d-appareils-connectes-dans-le-monde--2020/

[17] Enerdata, "World Energy Production," https://yearbook.enerdata.net/total-energy/world-energy-production.html, 2020.

[18] O. Bonnaud and A. Bsiesy, "Adaptation of the Higher Education in Engineering to the Advanced Manufacturing Technologies," Advances in Technology Innovation, vol. 5, no. 2, pp. 65-75, April 2020.

[19] O. Bonnaud, "Knowledge and Know-How in Microelectronics; Strategy of Innovative Practice to Balance the New On-line Courses Approach," International Conference on Advances in Technology Engineering, June 2017, pp. 10-11.

[20] O. Bonnaud and L. Fesquet, "Communicating and Smart Objects: Multidisciplinary Topics for the Innovative Education in Microelectronics and its Applications," 15th International Conference Information Technology Based Higher Education and Training, IEEE Press, August 2015, pp. 1-5.

[21] CNFM: "Coordination Nationale Pour La Formation En Microélectronique and Nanotechnologies," www.cnfm.fr, December 2020.

[22] O. Bonnaud, P. Gentil, A. Bsiesy, S. Retailleau, E. D. Gergam, and J. M. Dorkel, "GIP-CNFM: A French Education Network Moving from Microelectronics to Nanotechnologies," IEEE Global Engineering Education Conference, April 2011, pp. 122-127. 
[23] ACSIEL Alliance Électronique, "Components and Systems Alliance for Electronics Industry in France," http://www.acsiel.fr/en-GB/index.aspx, 2019.

[24] B. Pradarelli, O. Bonnaud, P. Nouet, and P. Benoit, "CNFM: Innovative Single National Entry-Point for Lifelong Learning in Microelectronics," Proceedings of EDULEARN, July 2019, pp. 1-3.

[25] FINMINA, "Formations Innovantes en Microélectronique et Nanotechnologies," http://www.cnfm.fr/?q=fr/presentation_FINMINA, March 2020.

[26] O. Bonnaud, "FINMINA: A French National Project Dedicated to Educational Innovation in Microelectronics to Meet the Challenges of a Digital Society," Smart Innovation, Systems and Technologies, vol. 188, pp. 31-44, June 2020.

[27] O. Bonnaud and L. Fesquet, "Practice in microelectronics education as a mandatory supplement to the future digital-based pedagogy: Strategy of the French national network," 2016 11th European Workshop on Microelectronics Education (EWME), Southampton, 2016, pp. 1-6.

[28] O. Bonnaud, "New Vision in Microelectronics Education: Smart E-Learning and Know-How, A Complementary Approach,” Smart Innovation, Systems and Technologies, vol. 99, pp. 267-275, May 2018.

[29] O. Bonnaud, "Introduction of the Multidisciplinary Know-How of the French National Training Network to Contribute to the Evolution of Microelectronic Technologies Towards Connected Objects," 17th International Conference on Information Technology Based Higher Education and Training (ITHET), August 2018, pp. 1-4.

[30] O. Bonnaud, "The Multidisciplinary Approach: A Common Trend for ULSI and Thin Film Technology," ECS Transaction, vol. 67, no. 1, pp. 147-158, 2015.

[31] O. Bonnaud and L. Fesquet, "Innovation in Higher Education: Specificity of the Microelectronics Field," 31st Symposium on Microelectronics Technology and Devices, IEEE Press, September 2016, pp. 119-122.

[32] O. Bonnaud and L. Wei, "A Way to Introduce Innovative Approach in the Field of Microelectronics and Nanotechnologies in the Chinese Education System," Proceedings of Engineering and Technology Innovation, vol. 4, pp. 19-21, October 2016.

[33] O. Bonnaud, Y. Danto, Y. H. Kuang, and L. Yuan, "International Flipped Class for Chinese Honors Bachelor Students in the Frame of Multidisciplinary Fields: Reliability and Microelectronics,” Advances in Technology Innovation, vol. 3, no. 3, pp. 126-132, March 2018.

Copyright $\odot$ by the authors. Licensee TAETI, Taiwan. This article is an open access article distributed under the terms and conditions of the Creative Commons Attribution (CC BY-NC) license (https://creativecommons.org/licenses/by-nc/4.0/). 